\title{
Biogeochemical evidence of anaerobic methane oxidation on active sub- marine mud volcanoes on the continental slope of the Canadian Beaufort Sea
}

\section{Dong-Hun Lee et al.}

Correspondence to: Jung-Hyun Kim (jhkim123@kopri.re.kr) and Kyung-Hoon Shin (shinkh@ hanyang.ac.kr)

The copyright of individual parts of the supplement might differ from the CC BY 4.0 License. 


\section{Supplementary information}

2 Table S1. Summary of pyrosequencing reads.

3

\begin{tabular}{|c|c|c|c|c|c|c|c|c|c|}
\hline \multirow[b]{2}{*}{ Sites } & \multirow{2}{*}{$\begin{array}{c}\text { Core } \\
\text { depth } \\
\text { (mbsf) }\end{array}$} & \multicolumn{5}{|c|}{ Summary of SSU rRNA tags } & \multicolumn{3}{|c|}{ Diversity index } \\
\hline & & $\begin{array}{l}\text { Number of total } \\
\text { reads }\end{array}$ & Archaeal reads & Bacterial reads & Eukaryotic reads & Unknown reads & Shannon & Simson & Ace \\
\hline \multirow{10}{*}{ MV282 } & 0.02 & 12214 & 8835 & 134 & 2790 & 455 & 1.53 & 0.34 & 36.34 \\
\hline & 0.09 & 8875 & 7016 & 36 & 1652 & 171 & 1.60 & 0.33 & 187.09 \\
\hline & 0.20 & 8222 & 8060 & 24 & 53 & 85 & 1.56 & 0.28 & 33.15 \\
\hline & 0.33 & 7304 & 7224 & 5 & 34 & 41 & 1.05 & 0.44 & 40.90 \\
\hline & 0.50 & 6182 & 6157 & 9 & 9 & 7 & 1.11 & 0.42 & 144.99 \\
\hline & 0.88 & 8886 & 8780 & 13 & 32 & 61 & 0.96 & 0.46 & 24.14 \\
\hline & 1.05 & 6283 & 6266 & 0 & 15 & 2 & 1.03 & 0.45 & 24.93 \\
\hline & 1.30 & 5058 & 5005 & 6 & 10 & 37 & 1.06 & 0.44 & 33.37 \\
\hline & 1.60 & 1902 & 1875 & 2 & 9 & 16 & 1.33 & 0.38 & 34.30 \\
\hline & 1.90 & 3550 & 3542 & 0 & 4 & 4 & 0.92 & 0.51 & 131.86 \\
\hline \multirow{10}{*}{ MV420 } & 0.08 & 3155 & 3028 & 23 & 29 & 75 & 1.28 & 0.55 & 104.50 \\
\hline & 0.20 & 4189 & 4079 & 22 & 32 & 56 & 2.68 & 0.12 & 125.60 \\
\hline & 0.33 & 5164 & 1436 & 64 & 3508 & 156 & 2.84 & 0.11 & 68.23 \\
\hline & 0.50 & 2175 & 2041 & 1 & 44 & 89 & 2.49 & 0.15 & 133.00 \\
\hline & 0.70 & 2307 & 2259 & 1 & 3 & 44 & 2.08 & 0.24 & 74.00 \\
\hline & 1.05 & 1537 & 1520 & 0 & 10 & 7 & 1.47 & 0.44 & 102.82 \\
\hline & 1.38 & 5207 & 4757 & 71 & 107 & 272 & 1.74 & 0.35 & 40.90 \\
\hline & 1.60 & 7012 & 6985 & 6 & 8 & 13 & 1.45 & 0.42 & 37.70 \\
\hline & 1.81 & 3706 & 3669 & 5 & 7 & 25 & 1.40 & 0.45 & 52.58 \\
\hline & 2.17 & 12017 & 11865 & 11 & 80 & 61 & 1.36 & 0.47 & 75.61 \\
\hline \multirow{5}{*}{ MV740 } & 0.08 & 522 & 445 & 17 & 29 & 31 & 3.59 & 0.05 & 178.09 \\
\hline & 0.20 & 506 & 458 & 0 & 28 & 20 & 3.04 & 0.11 & 170.50 \\
\hline & 0.35 & 674 & 589 & 1 & 75 & 9 & 3.12 & 0.09 & 112.57 \\
\hline & 0.45 & 583 & 534 & 0 & 35 & 14 & 2.84 & 0.10 & 82.31 \\
\hline & 0.55 & 706 & 673 & 1 & 6 & 26 & 2.92 & 0.09 & 97.31 \\
\hline
\end{tabular}


5 Table S2. Heat map and taxonomic affiliation three dominant methanomicrobial OTUs along the depth. The color gradient from white to brown

6 indicates low to high relative abundance values.

7

\begin{tabular}{|c|c|c|c|c|c|c|c|c|c|c|c|c|c|c|c|c|c|c|c|c|c|c|c|c|c|c|c|c|c|c|c|}
\hline \multirow{3}{*}{ OTU_ID } & \multicolumn{6}{|c|}{ Taxonomy } & \multirow{2}{*}{\multicolumn{10}{|c|}{\begin{tabular}{|l|} 
MV282 \\
Core depth (mbsf) \\
\end{tabular}}} & \multirow{2}{*}{\multicolumn{10}{|c|}{$\begin{array}{l}\text { MV420 } \\
\text { Core depth (mbsf) }\end{array}$}} & \multirow{2}{*}{\multicolumn{5}{|c|}{$\begin{array}{l}\text { MV740 } \\
\text { Core depth (mbsf) }\end{array}$}} \\
\hline & \multirow{2}{*}{ phylum } & \multirow{2}{*}{ class } & \multirow{2}{*}{ order } & \multirow{2}{*}{ family } & \multirow{2}{*}{ genus } & \multirow{2}{*}{ species } & & & & & & & & & & & & & & & & & & & & & & & & & \\
\hline & & & & & & & 0.02 & 0.09 & 0.20 & 0.33 & 0.50 & 0.88 & 1.05 & 1.30 & 1.60 & 1.90 & 0.08 & 0.20 & 0.33 & 0.50 & 0.70 & 1.05 & 1.38 & 1.60 & 1.81 & 2.17 & 0.08 & 0.20 & 0.35 & 0.45 & 0.55 \\
\hline c116 & Euryarchaeota & Methanomicrobia & Methanosarcinales & ANME3_f & ANME3_g & ANME3_S & 2.5 & 2.0 & 14.1 & 0.0 & 0.0 & 0.0 & 0.0 & 0.0 & 0.0 & 0.1 & 5.5 & 3.4 & 6.1 & 0.2 & 6.7 & 0.5 & 0.0 & 0.0 & 0.0 & 0.0 & 0.0 & 0.2 & 0.0 & 0.0 & 0.0 \\
\hline c1698 & Euryarchaeota & Methanomicrobia & Methanosarcinales & ANME3 $\_f$ & ANME3_g & ANME3_S & 1.3 & 0.9 & 0.0 & 0.0 & 0.0 & 0.0 & 0.0 & 0.0 & 0.0 & 0.0 & 0.9 & 0.3 & 0.4 & 0.0 & 0.0 & 0.0 & 0.0 & 0.0 & 0.0 & 0.0 & 0.0 & 0.0 & 0.0 & 0.0 & 0.0 \\
\hline c1784 & Euryarchaeota & Methanomicrobia & Methanosarcinales & ANME2 $f$ & ANME2c $\_g$ & DQ084449_s & 1.7 & 3.4 & 6.8 & 1.2 & 0.0 & 0.0 & 0.1 & 0.0 & 0.0 & 0.7 & 0.0 & 0.0 & 0.1 & 0.9 & 1.3 & 0.3 & 0.0 & 0.0 & 0.1 & 0.1 & 0.0 & 0.7 & 3.7 & 11.2 & 14.9 \\
\hline
\end{tabular}

8 
10 Fig. S1. Examples of GC-MS chromatograms of polar fractions obtained from sediment cores

11 (A) ARA05C-10-GC (MV282): core depth 0.1 m, (B) ARA05C-01-GC (MV420): core depth

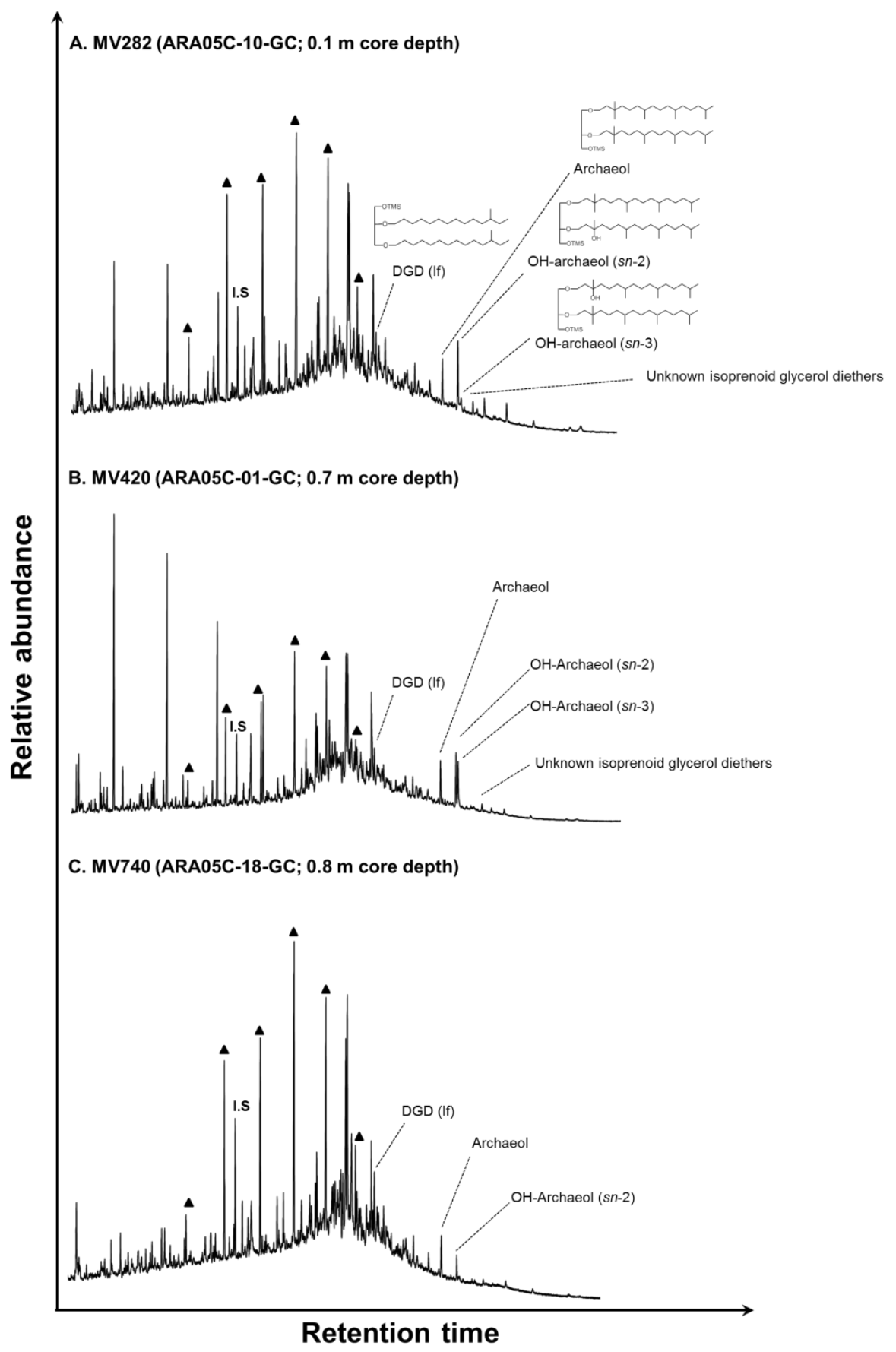


14 Fig. S2. Relative abundances of archaeal communities at the class level along the depth (A)

15 MV282, (B) MV420 and (C) MV740.

\section{Relative abundance of $16 \mathrm{~S}$ rRNA sequences (\%)}
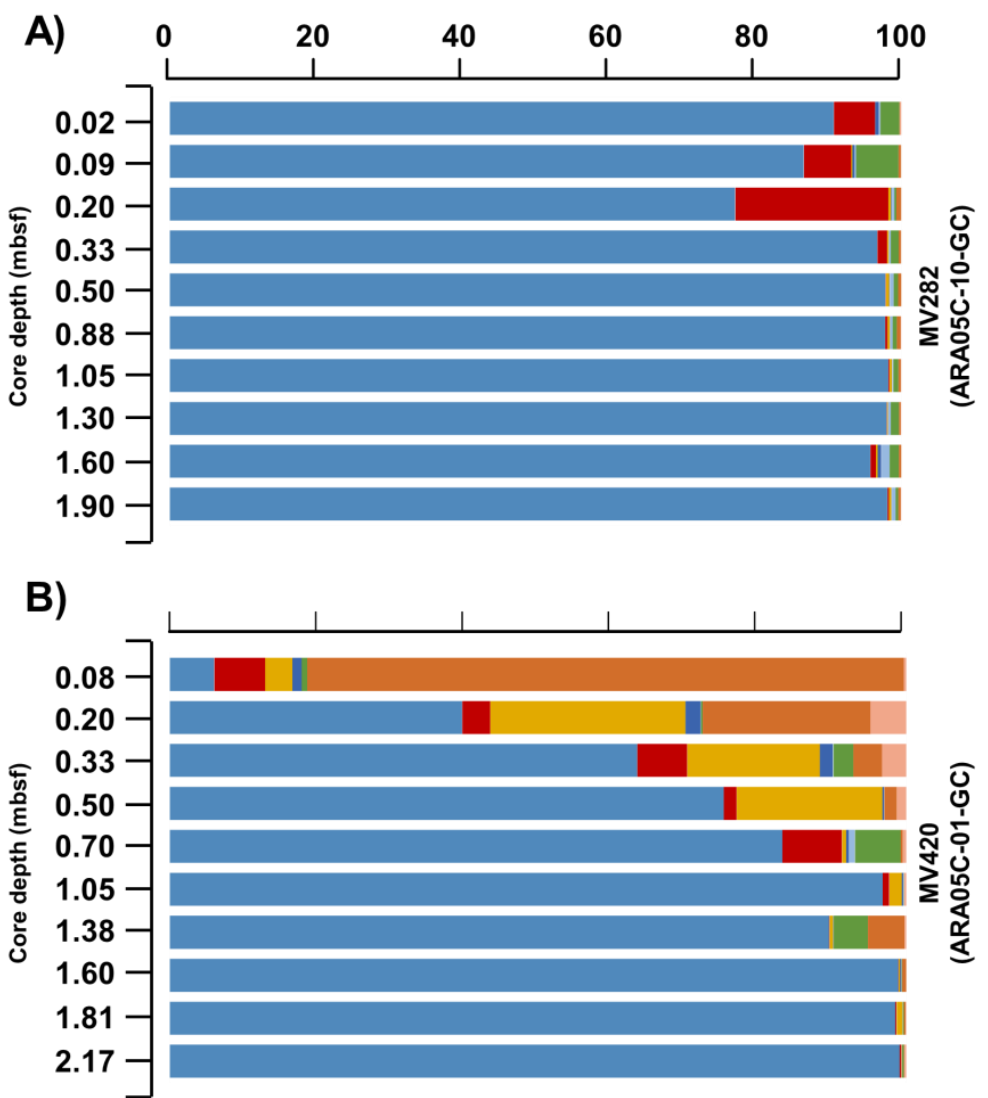

C)

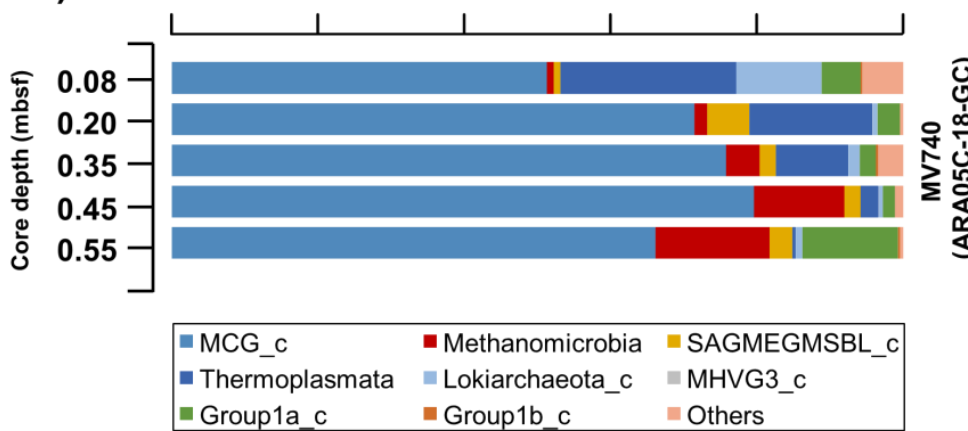

